\title{
Referral of patients from Lithuania to determination of dehydroepiandrosterone sulphate and no clinical consequences of results
}

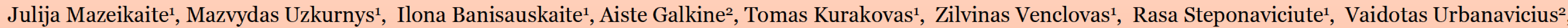
Rasa Verkauskiene', Valentinas Matulevicius ${ }^{1}$.

${ }^{1}$ Institute of Endocrinology, Lithuanian University of Health Sciences, Kaunas, Lithuania

2 Vilnius University, Faculty of Medicine, Endocrinology Center

\section{Introduction}

Recently Matulevicius et al ${ }^{1}$ found the case of a thirty-eight-year-old woman with complaints of secondary amenorrhea, hirsutism, decreased sexuality, weight gain and epileptic seizures. This patient also presented with elevated dehydroepiandrosterone sulphate(DHEAS) and testosterone levels. According to these symptoms we performed a retrospective study to see if there were more cases of dehoma (elevation of DHEAS + adrenal tumor).

\section{Conclusion}

The clinical features of the patients with high DHEAS reveals increase of body weight, BMI and hirsutism in women but increase of elevated blood pressure and frequency of adrenal tumor in men.

\section{Methods}

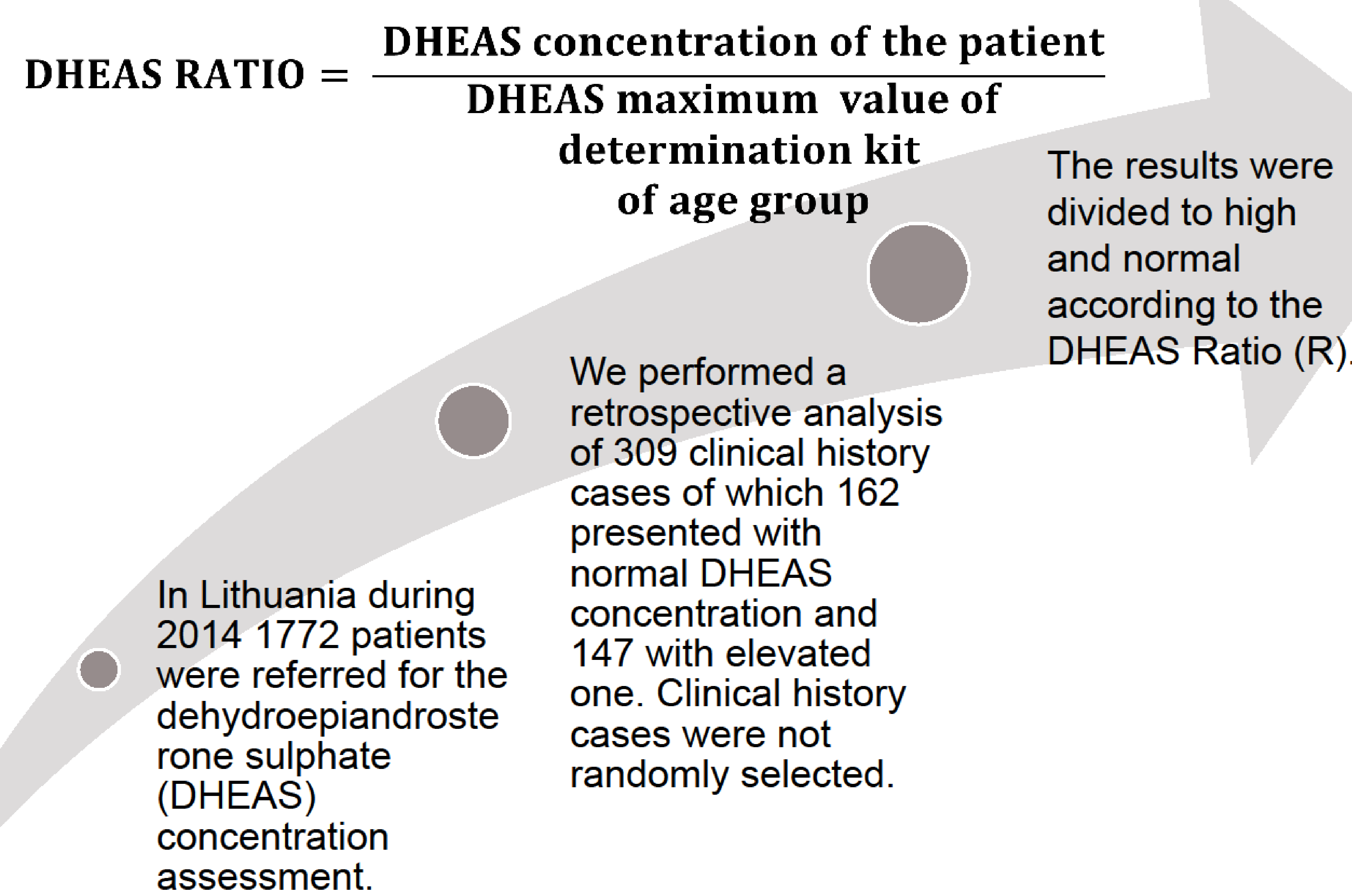

\begin{tabular}{cccc} 
& DHEAS & $\begin{array}{c}\text { DHEAS } \\
\text { Ratio }\end{array}$ & Testosterone \\
\hline $\begin{array}{c}\text { Healthy } \\
\text { women }\end{array}$ & $5.7 \pm 3.05$ & $0.54 \pm 0.23$ & $3.29 \pm 1.36$ \\
$\begin{array}{c}\uparrow \uparrow \uparrow \\
\text { DHEAS }\end{array}$ & $12.49 \pm 3.81$ & $1.3 \pm 0.35$ & $4.27 \pm 1.83$ \\
\hline p & $<0.001$ & $<0.001$ & $<0.00001$ \\
\hline
\end{tabular}

Table 1 . Concentration of DHEAS and testosterone of women.

\begin{tabular}{|c|c|c|c|c|c|c|c|c|}
\hline Parameter & $\mathbf{n}$ & Hirsutism & $\begin{array}{l}\text { Menstruation } \\
\text { cycle } \\
\text { disorder }\end{array}$ & $\begin{array}{l}\text { Weight } \\
\text { gain }\end{array}$ & Infertility & $\begin{array}{l}\text { Elevated blood } \\
\text { pressure }\end{array}$ & $\begin{array}{c}\text { Body } \\
\text { weight, kg } \\
\text { (Mean士SD) }\end{array}$ & $\begin{array}{c}n \\
\text { (different } \\
\text { items) }\end{array}$ \\
\hline Normal & 142 & $21.7 \%$ & $32.3 \%$ & - & $10.6 \%$ & $8.1 \%$ & $72.54 \pm 22.41$ & 20 \\
\hline $\begin{array}{c}\uparrow \uparrow \uparrow \\
\text { DHEAS }\end{array}$ & 123 & $39 \%$ & $23 \%$ & $8 \%$ & - & - & $80.72 \pm 20.85$ & 12 \\
\hline
\end{tabular}

Table 2. The complaints of female patients.

$30 \%$
$25 \%$
$20 \%$
$15 \%$
$5 \%$
$0 \%$ ultrasound $26.10 \%$

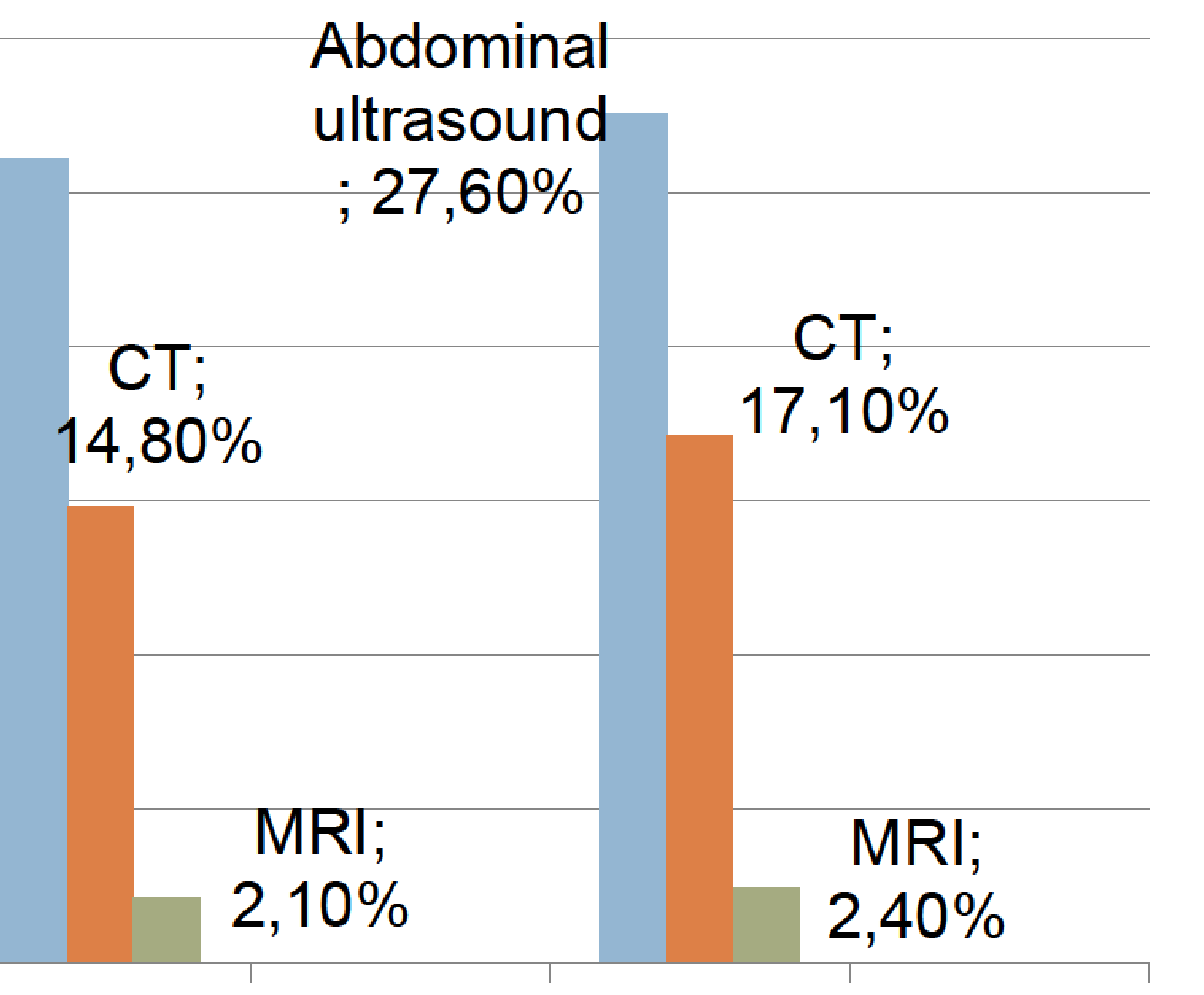

\begin{tabular}{|c|c|c|c|}
\hline & $\mathbf{n}$ & $\begin{array}{c}\uparrow \text { Blood } \\
\text { pressure }\end{array}$ & $\begin{array}{c}\text { Adrenal } \\
\text { tumor }\end{array}$ \\
\hline $\begin{array}{c}\text { Healthy } \\
\text { men }\end{array}$ & 20 & $10 \%$ & $25 \%$ \\
\hline $\begin{array}{c}\uparrow \uparrow \uparrow \\
\text { DHEAS }\end{array}$ & 24 & $33 \%$ & $29.2 \%$ \\
\hline
\end{tabular}

Table 3 .The complaints of male patients.

Healthy women

$\uparrow \uparrow \uparrow$ DHEAS

\section{Results}

From the list of DHEA-S asessment in Lithuania during 1 year (2014) we selected cases with normal and elevated DHEA-S.

In women with high DHEA-S, weight, body mass index and hirsutism were found statistically higher as compared with healthy women. No difference in menstrual cycle disorder was observed. In men, there was a clinical difference: in patients with high DHEAS, higher blood pressure was found as well as frequency of adrenal tumors.

Chart 1.The percentage of diagnostic imaging of women.

\section{References}

${ }^{1}$ Matulevicius V, Ostrauskas R, Krasauskas V, Verkauskiene R, Ciaplinskiene L, Urbanavicius V. ADRENAL ANDROGEN PRODUCING ADENOMA ASSOCIATED WITH EPILEPTIC SEIZURES. Acta Endocrinologica; 2014;10(3):p487 